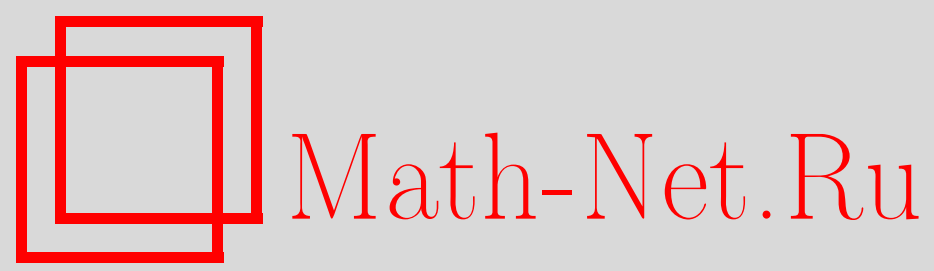

А. А. Паньков, Упругие волны в композитах со случайными структурами, Вестн. Сам. гос. техн. ун-та. Сер. Физ.-мат. науки, 2001, выпуск 12, 85-90

DOI: https://doi.org/10.14498/vsgtu65

Использование Общероссийского математического портала Math-Net.Ru подразумевает, что вы прочитали и согласны с пользовательским соглашением

http://www.mathnet.ru/rus/agreement

Параметры загрузки:

IP: 54.162 .85 .209

26 апреля 2023 г., 17:44:02 


\title{
А.А.Паньков
}

\section{УПРУГИЕ ВОЛНЫ В КОМПОЗИТАХ СО СЛУЧАЙНЫМИ СТРУКТУРАМИ}

\begin{abstract}
Представлены решения для динамических эффективных упругих и дифракционных свойств композитов со случайныли структурами из однородных включений, полученные на основе решения соответствующих осредненных волновых уравнений обобщенного метода самосогласования. Расчетная схема осредненной задачи - дифракция продольной и поперечной волн, падающих на расположенное в среде с искомыли динамическими эффективными упругими свойствами одиночное включение, окруженное переходным слоем с неоднородными упругими свойствами. Приведены результаты численных расчетов компонент тензора динамических эффективных упругих свойств и сечений рассеяния для однонаправленного волокнистого композита в плоскости изотропии при различных значениях круговой частоты.
\end{abstract}

В задачах о распространении волн в композитах характерный размер неоднородностей имеет определяющее значение и динамическое поведение композитов можно, в наиболее общем случае, отнести к одному из двух видов. В первом длина волны значительно больше характерного масштаба неоднородности композита и поведение композита описывается через соответствующие статические эффективные физико-механические свойства. Ко второму виду относится поведение композита, когда длина волны соизмерима с характерным масштабом неоднородности и в связи с чем в композите могут возникать очень сложные динамические эффекты, так как наличие поверхностей раздела фаз в композите приводит к возникновению отраженных и преломленных волн. Одной из актуальных задач механики композитов является исследование распространения и анализ эффекта затухания волн в матричных композитах с учетом дифракции волн на случайным образом расположенных в матрице композита включениях. Понятие элементарного объема $\mathbf{V}$ в динамике рассеивающих сред [1-8] вводится как объем, в котором количество поглощенной и рассеянной энергии волн пропорционально его размеру. Для этого необходимо, чтобы он был, с одной стороны, достаточно велик, т.е. чтобы статистические характеристики распространения волн были близки к средним, а с другой - мал, чтобы интенсивность падающей на него волны оставалась почти неизменной в его пределах [3].

Будем рассматривать распространение и эффект затухания гармонических волн в матричных композитах, например, когда все включения имеют одинаковые геометрическую форму, ориентацию и случайный размер. Упругие свойства и плотности включений и матрицы композита различны. Выполняются условия идеального контакта на межфазных поверхностях. Величины относящиеся к включениям и матрице будем обозначать индексами $F$ и $M$ соответственно.

В области $\mathbf{V}$ на микроуровне композита уравнения движения

$$
\sigma_{i j, j}(\mathbf{r}, t)=\rho(\mathbf{r}) \frac{\partial^{2} u_{i}(\mathbf{r}, t)}{\partial t^{2}}
$$

с учетом разложений

$$
u_{i}(\mathbf{r}, t)=u_{(a) i}(\mathbf{r}) \mathrm{e}^{-i \omega t}, \quad \sigma_{i j}(\mathbf{r}, t)=\sigma_{(a) i j}(\mathbf{r}) \mathrm{e}^{-i \omega t}
$$

принимают вид

$$
\sigma_{(a) i j, j}(\mathbf{r})-\rho(\mathbf{r}) \omega^{2} u_{(a) i}(\mathbf{r})=0
$$

или

$$
\left(C_{i j m n}(\mathbf{r}) u_{(a) m, n}(\mathbf{r})\right)_{, j}+\rho(\mathbf{r}) \omega^{2} u_{(a) i}(\mathbf{r})=0
$$

относительно комплексных амплитуд смещений $\mathbf{u}_{(a)}(\mathbf{r})$ гармонических колебаний; в (1)-(3) $\omega$ - круговая частота. Поля упругих свойств $\mathbf{C}(\mathbf{r})$ и плотности $\rho(\mathbf{r})$ в области $\mathbf{V}$ имеют вид:

$$
\begin{array}{r}
C_{i j m n}(\mathbf{r})=\omega_{F}(\mathbf{r}) C_{i j m n}^{F}+\left(1-\omega_{F}(\mathbf{r})\right) C_{i j m n}^{M}, \\
\rho(\mathbf{r})=\omega_{F}(\mathbf{r}) \rho_{F}+\left(1-\omega_{F}(\mathbf{r})\right) \rho_{M},
\end{array}
$$

где $\mathbf{C}^{F}, \rho_{F}$ и $\mathbf{C}^{M}, \rho_{M}$ - тензоры упругих свойств и величины плотностей включений и матрицы композита соответственно, индикаторная функция включений 


$$
\omega_{F}(\mathbf{r})=\left\{\begin{array}{ll}
1, & \mathbf{r} \in \mathbf{V}_{F} \\
0, & \mathbf{r} \notin \mathbf{V}_{F}
\end{array},\right.
$$

где $\mathbf{V}_{F}$ - область включений в $\mathbf{V}$. Величина $v_{F} \equiv<\omega_{F}>$ - относительное объемное содержание включений в композите, где оператор статистического осреднения <...> для исследуемых статистически однородных и эргодических случайных полей совпадает с оператором объемного осреднения [6]:

$$
<\ldots>=\frac{1}{|\mathbf{V}|} \int_{\mathbf{V}} \ldots d \mathbf{r} .
$$

Случайный разброс относительных размеров включений в композите определим через представительную выборку коэффициентов подобия $\left\{\alpha_{(k)} ; k=1, N\right\}$ этих размеров относительно нормированных размеров некоторого формального включения $\mathbf{v}$, где $N$ - число включений в области $\mathbf{V}$. Размеры формального включения $\mathbf{V}$ определим из условия

$$
|\mathbf{v}| \equiv \frac{1}{N} \sum_{k=1}^{N}\left|\mathbf{v}_{(k)}\right|
$$

связывающего объем включения $|\mathbf{v}|$ с совокупностью значений объемов $\left\{\left|\mathbf{v}_{(k)}\right| ; k=\overline{1, N}\right\},\left|\mathbf{v}_{(k)}\right|$ - объем $k$-го включения в области $\mathbf{V}$. В результате для коэффициентов подобия имеем равенство

$$
\frac{1}{N} \sum_{k=1}^{N} \alpha_{(k)}^{\beta}=1
$$

так как выполняется зависимость $\left|\mathbf{v}_{(k)}\right|=\alpha_{(k)}^{\beta}|\mathbf{v}|$, где показатель степени $\beta$ связан с размерностью задачи и равен 1, 2 или 3 для слоистого, однонаправленного волокнистого и гранулированного композитов соответственно.

Рассмотрим вычисление тензора динамических эффективных упругих свойств $\mathbf{C}^{*}$ композита, входящего в обобщенный закон Гука на макроуровне композита

$$
\sigma_{(a) i j}^{*}(\mathbf{r})=\mathrm{C}_{i j m n}^{*} \varepsilon_{(a) m n}^{*}(\mathbf{r})
$$

и связывающего амплитуды макронапряжений и макродеформаций

$$
\sigma_{(a) i j}^{*}=\left\langle\sigma_{(a) i j}(\mathbf{r})\right\rangle, \quad \quad \varepsilon_{(a) i j}^{*}=\left\langle\varepsilon_{(a) i j}(\mathbf{r})\right\rangle .
$$

Тензор динамических эффективных упругих свойств композита $\mathbf{C}^{*}$ может быть рассчитан по формуле

$$
C_{i j m n}^{*}=C_{i j m n}^{M}+v_{F}\left(C_{i j p q}^{F}-C_{i j p q}^{M}\right) N_{(a) p q m n}^{F},
$$

где $\mathbf{N}_{(a)}^{F}$ - тензоры концентраций осредненных комплексных амплитуд деформаций на включениях композита, которые вводятся разложением

$$
\left.\varepsilon_{(\mathrm{a}) i j}^{F} \equiv<\varepsilon_{(a) i j}(\mathbf{r})\right\rangle_{F}=N_{(a) i j m n}^{F} \varepsilon_{(a) m n}^{*},
$$

где $\langle\ldots\rangle_{F}$ - оператор объемного осреднения по области включений композита $\mathbf{V}_{F}$ аналогично оператору (7).

Таким образом, задача расчета тензора динамических эффективных упругих свойств $\mathbf{C}^{*}$ композита сводится к расчету тензоров концентраций осредненных комплексных амплитуд деформаций на включениях $\mathbf{N}_{(a)}^{F}$. Рассмотрим вычисление тензоров $\mathbf{N}_{(a)}^{F}$ из решения соответствующих осредненных задач обобщенного метода самосогласования $[9,10]$.

Постановка осредненных задач в локальной системе координат. В локальной системе координат, начало которой совмещено с центром произвольного $k$-го включения из области $\mathbf{V}$, введем в рассмотрение осредненные поля $g$-го порядка для комплексных амплитуд перемещений, деформаций и напряжений:

$$
\bar{u}_{(a) i}^{(g)}(\xi) \equiv \frac{1}{N} \sum_{k=1}^{N} \alpha_{(k)}^{\beta+g-1} u_{(a) i}\left(\mathbf{r}_{(k)}+\alpha_{(k)} \xi\right),
$$




$$
\begin{aligned}
& \bar{\varepsilon}_{(a) i j}^{(g)}(\xi) \equiv \frac{1}{N} \sum_{k=1}^{N} \alpha_{(k)}^{\beta+g} \varepsilon_{(a) i j}\left(\mathbf{r}_{(k)}+\alpha_{(k)} \xi\right)=\bar{u}_{(a)(i, j)}^{(g)}(\xi), \\
& \bar{\sigma}_{(a) i j}^{(g)}(\xi) \equiv \frac{1}{N} \sum_{k=1}^{N} \alpha_{(k)}^{\beta+g} \sigma_{(a) i j}\left(\mathbf{r}_{(k)}+\alpha_{(k)} \xi\right) .
\end{aligned}
$$

Для значений $g=0,1,2, \ldots$, где $\mathbf{r}_{(k)}$ - радиус-вектор центра $k$-го включения в области $\mathbf{V}$, индексы $i$ и $j$ в круглых скобках обозначают операцию выделения симметричной составляющей из поля градиентов осредненных перемещений [6].

Искомые значения компонент тензоров

$$
\mathbf{N}_{(\mathrm{a})}^{F} \equiv \mathbf{N}_{(\mathrm{a})}^{F(0)}
$$

могут быть рассчитаны через осредненные поля амплитуд перемещений $\overline{\mathbf{u}}_{(a)}^{(0)}(\xi)$ или амплитуд деформаций $\bar{\varepsilon}_{(a) i j}^{(0)}(\xi) \equiv \bar{u}_{(a)(i, j)}^{(0)}(\xi)(13)$, например, по формуле

$$
\frac{1}{|\mathbf{v}|} \int_{\mathbf{v}} \bar{\varepsilon}_{(a) i j}^{(0)}(\xi) d \xi=N_{(a) i j m n}^{F(0)} \varepsilon_{(a) m n}^{*},
$$

При этом центр нормированного формального включения $\mathbf{v}$ совмещен с началом локальной системы координат $\xi$.

Осредненные поля (13) возможно определить из решения соответствующих осредненных волновых уравнений

$$
\frac{\partial}{\partial \xi_{j}}\left(\bar{a}_{i j m n}^{(g)}(\xi) \frac{\partial}{\partial \xi_{n}} \bar{u}_{(a) m}^{(g)}(\xi)\right)+\omega^{2} \bar{\rho}_{i j}^{(g)}(\xi) \bar{u}_{(a) j}^{(g)}(\xi)=0
$$

для различных значений $g=0,1,2, \ldots$. Тензорные поля приведенных плотностей в (16) имеют вид

$$
\bar{\rho}_{i j}^{(g)}(\xi) \equiv\left\{\begin{array}{cc}
\rho_{F} U_{(a) i p}^{F(g+2)} U_{(a) p j}^{F(g)-1}, & \xi \in \mathbf{v}, \\
{\left[\bar{\beta}^{(\beta+g+1)}(\xi) \rho_{M} \delta_{i p}+\left(\bar{\alpha}_{F}^{(\beta+g+1)}(\xi) \rho_{F}-\bar{\beta}^{(\beta+g+1)}(\xi) \rho_{M}\right) v_{F} U_{(a) i p}^{F(1)}\right] s_{p j}^{(g)-1}(\xi),} & \xi \notin \mathbf{v},
\end{array}\right.
$$

где для $\xi \notin \mathbf{v}$ тензор $\mathbf{s}^{(g)-1}$ обратный тензору

$$
s_{i j}^{(g)}(\xi) \equiv \bar{\beta}^{(\beta+g-1)}(\xi) \delta_{i p}+\left(\bar{\alpha}_{F}^{(\beta+g-1)}(\xi)-\bar{\beta}^{(\beta+g-1)}(\xi)\right) v_{F} U_{(a) i p}^{F(1)},
$$

символы Кронекера $\delta_{i j}=\left\{\begin{array}{ll}1, & i=j \\ 0, & i \neq j\end{array}\right.$. Поля приведенных упругих свойств в (16):

$$
\bar{a}_{i j m n}^{(g)}(\xi)=\left\{\begin{array}{cl}
C_{i j m n}^{F}, & \xi \in \mathbf{v}, \\
{\left[\bar{\beta}^{(\beta+g)}(\xi) C_{i j q p}^{M}+\left(\bar{\alpha}_{F}^{(\beta+g)}(\xi) C_{i j d b}^{F}-\bar{\beta}^{(\beta+g)}(\xi) C_{i j d b}^{M}\right) v_{F} N_{(a) d b q p}^{F(0)}\right] k_{q p m n}^{(g)-1}(\xi),} & \xi \notin \mathbf{v},
\end{array}\right.
$$

где для $\xi \notin \mathbf{v}$ тензор $\mathbf{k}^{(g)-1}$ обратный тензору

$$
k_{i j m n}^{(g)}(\xi) \equiv \bar{\beta}^{(\beta+g)}(\xi) E_{i j m n}+\left(\bar{\alpha}_{F}^{(\beta+g)}(\xi)-\bar{\beta}^{(\beta+g)}(\xi)\right) v_{0} N_{(a) i j m n}^{F(0)},
$$

$E_{i j m n}=\frac{1}{2}\left(\delta_{i m} \delta_{j n}+\delta_{i n} \delta_{j m}\right)$ - единичный тензор. Структурные функции в (17)-(20)

$$
\bar{\alpha}_{F}^{(p)}(\xi) \equiv \frac{\bar{\omega}_{F}^{(p)}(\xi)}{v_{F}}, \quad \quad \bar{\beta}^{(p)}(\xi) \equiv \frac{\eta_{(p)}-\bar{\omega}_{F}^{(p)}(\xi)}{1-v_{F}}
$$

рассчитываются через осредненную индикаторную функцию $p$-го порядка

$$
\bar{\omega}_{F}^{(p)}(\xi) \equiv \frac{1}{N} \sum_{k=1}^{N} \alpha_{(k)}^{p} \omega_{F}\left(\mathbf{r}_{(k)}+\alpha_{(k)} \xi\right)
$$

и учитывают особенности случайной структуры композита через заданное поле $\omega_{F}(\mathbf{r})(6)$. При значениях $|\xi|$, превышающих радиус корреляции случайной структуры композита, например, при $|\xi| \rightarrow \infty$, выполняется равенство:

$$
\bar{\omega}_{F}^{(p)}=\eta_{(p)} v_{F}
$$

для осредненных индикаторных функций (22) и 


$$
\bar{u}_{(a) i}^{(g)}=\eta_{(\beta+g)} u_{(a) i}^{*}, \bar{\varepsilon}_{(a) i j}^{(g)}=\eta_{(\beta+g)} \varepsilon_{(a) i j}^{*}, \bar{\sigma}_{(a) i j}^{(g)}=\eta_{(\beta+g)} \sigma_{(a) i j}^{*}
$$

для осредненных полей деформирования (13), где структурные коэффициенты

$$
\eta_{(p)} \equiv \frac{1}{N} \sum_{k=1}^{N} \alpha_{(k)}^{p}
$$

при этом в частном случае при $p=\beta$ имеем $\eta_{(\beta)} \equiv 1$ с учетом равенств (8) и (25).

Тензоры $\mathbf{U}_{(a)}^{F(g)}$ в (17) и (18), аналогично тензорам $\mathbf{N}_{(a)}^{F(0)}(15)$, вводятся разложением осредненных по включениям композита амплитуд перемещений

$$
u_{(a) i}^{F(g)} \equiv \frac{1}{|\mathbf{v}|} \int_{\mathbf{v}} \bar{u}_{(a) i}^{(g)}(\xi) d \xi=U_{(a) i j}^{F(g)} u_{(a) j}^{*}
$$

и рассчитываются через поле $\overline{\mathbf{u}}_{(a)}^{(g)}(\xi)(2.1)$.

В результате приходим к системе (16) из бесконечной цепочки зацепляющихся осредненных волновых уравнений. Если ограничиться рассмотрением лишь первых $H+1$ уравнений, тогда встает проблема замыкания этой новой системы. Для этого используем подход, аппробированный ранее в работе [10]. Воспользуемся предположением об однородности комплексных амплитуд перемещений в пределах $k$-го включения $\mathbf{u}_{(a)}^{F(k)}$ в области композита $\mathbf{V}$ и аппроксимацией их суммой, например, степенного ряда

$$
u_{(a) i}^{F(k)}=\sum_{g=0}^{H} \alpha_{(k)}^{-g} n_{i j}^{F(-g)} u_{(a) j}^{*} .
$$

Таким образом, система $H+1$ осредненных волновых уравнений (16) для значений $g=\overline{0, H}$ может быть дополнена недостающими зависимостями

$$
U_{(a) i j}^{F(g)}=\sum_{\tau=0}^{H} \eta_{(\beta+g-1-\tau)} n_{i j}^{F(-\tau)} .
$$

Частные случаи. Когда задан разброс размеров включений, тогда в осредненных волновых уравнениях (16) имеем

$$
\bar{\rho}_{i j}^{(g)}(\xi) \neq \rho,
$$

даже если плотности включений и матрицы композита равны между собой

$$
\rho_{F}=\rho_{M} \equiv \rho .
$$

Число $H+1$ осредненных волновых уравнений в системе (16) равно числу членов ряда в разложении (27). Например, пусть $H=1$, тогда с учетом (28) имеем

$$
\left\{\begin{array}{l}
\frac{\partial}{\partial \xi_{j}}\left(\bar{a}_{i j m n}^{(0)}(\xi) \frac{\partial}{\partial \xi_{n}} \bar{u}_{(a) m}^{(0)}(\xi)\right)+\omega^{2} \bar{\rho}_{i j}^{(0)}(\xi) \bar{u}_{(a) j}^{(0)}(\xi)=0, \\
\frac{\partial}{\partial \xi_{j}}\left(\bar{a}_{i j m n}^{(1)}(\xi) \frac{\partial}{\partial \xi_{n}} \bar{u}_{(a) m}^{(1)}(\xi)\right)+\omega^{2} \bar{\rho}_{i j}^{(1)}(\xi) \bar{u}_{(a) j}^{(1)}(\xi)=0, \\
U_{(a) i j}^{F(1)}=\eta_{(\beta)} n_{i j}^{F(0)}+\eta_{(\beta-1)} n_{i j}^{F(-1)}, \\
U_{(a) i j}^{F(2)}=\eta_{(\beta+1)} n_{i j}^{F(0)}+\eta_{(\beta)} n_{i j}^{F(-1)}, \\
U_{(a) i j}^{F(3)}=\eta_{(\beta+2)} n_{i j}^{F(0)}+\eta_{(\beta+1)} n_{i j}^{F(-1)},
\end{array}\right.
$$

где поля плотностей $\bar{\rho}^{(0)}(\xi)$ и $\bar{\rho}^{(1)}(\xi)$ рассчитываются по формулам (17) через тензоры $\mathbf{U}_{(a)}^{F(0)}$, $\mathbf{U}_{(a)}^{F(1)}, \mathbf{U}_{(a)}^{F(2)}$ и $\mathbf{U}_{(a)}^{F(3)}$, входящих в систему $(31)$; поля $\overline{\mathbf{a}}^{(0)}(\xi)$ и $\overline{\mathbf{a}}^{(1)}(\xi)$ рассчитываются через тензоры $\mathbf{N}_{(a)}^{F(0)}$ по формуле (19) с учетом равенства (15).

Когда плотности включений и матрицы композита различны и все включения одного размера, тогда выполняются равенства

$$
\alpha_{(k)}=1 \forall k=\overline{1, \quad N}
$$

и из системы осредненных волновых уравнений (31) остается лишь одно "нулевое" ( $g=0)$ волновое уравнение 


$$
\frac{\partial}{\partial \xi_{j}}\left(\bar{a}_{i j m n}^{(0)}(\xi) \frac{\partial}{\partial \xi_{n}} \bar{u}_{(a) m}^{(0)}(\xi)\right)+\omega^{2} \bar{\rho}_{i j}^{(0)}(\xi) \bar{u}_{(a) j}^{(0)}(\xi)=0,
$$

где поле плотностей (17) имеет вид

$$
\bar{\rho}_{i j}^{(0)}(\xi) \equiv\left\{\begin{array}{cc}
\rho_{F}, & \xi \in \mathbf{v} \\
{\left[\bar{\beta}^{(0)}(\xi) \rho_{M} \delta_{i p}+\left(\bar{\alpha}_{F}^{(0)}(\xi) \rho_{F}-\bar{\beta}^{(0)}(\xi) \rho_{M}\right) \nu_{0} U_{(a) i p}^{F(0)}\right] s_{p j}^{(0)-1}(\xi),} & \xi \notin \mathbf{v}
\end{array}\right.
$$

при этом для $\xi \notin \mathbf{v}$ тензор $\mathbf{s}^{(0)-1}$ обратный тензору

$$
s_{i j}^{(0)}(\xi) \equiv \bar{\beta}^{(0)}(\xi) \delta_{i p}+\left(\bar{\alpha}_{F}^{(0)}(\xi)-\bar{\beta}^{(0)}(\xi)\right) \nu_{F} U_{(a) i p}^{F(0)}
$$

с учетом равенства (18).

Однонаправленный волокнистый композит. Рассмотрим распространение продольных и поперечных гармонических волн в плоскости изотропии $r_{1} O r_{2}$ однонаправленного волокнистого композита в условиях плоской деформации (рис. 1). Считаем, что все волокна имеют одинаковые радиусы $r_{F}$ круглых поперечных сечений (32), центры которых статистически однородно распределены в узлах идеальной периодической гексагональной решетки с минимальной гарантированной прослойкой матрицы между волокнами в $4 \%$ от радиуса $r_{F}$. Пусть плотности волокон и матрицы совпадают (30). Осредненное волновое уравнение примет вид (33), где с учетом (30) надо положить $\rho^{(0)}(\xi)=\rho$. Расчетная схема дифракция продольной и поперечной волн, падающих на расположенное в среде с искомыми динамическими эффективными упругими свойствами $\mathbf{C}^{*}$ одиночное включение $\mathbf{v}$, окруженное переходным слоем с неоднородными приведенными упругими свойствами $\overline{\mathbf{a}}^{(0)}(\xi)$.

Для численного решения осредненной задачи использована полярная система координат



Р и с. 1. Представительная область композита в плоскости изотропии

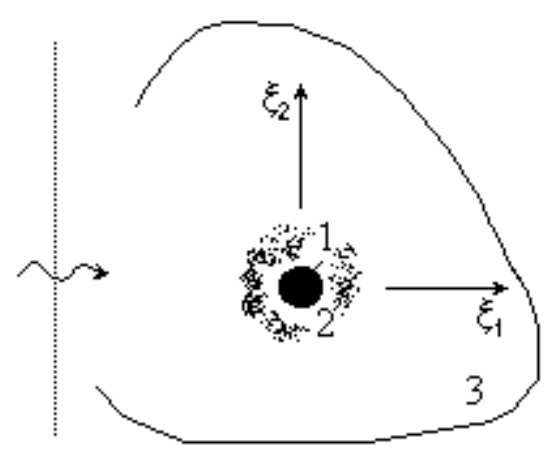

Р и с. 2. Расчетная схема обобщенного метода самосогласования: 1 - волокно, 2 - неоднородная окрестность, 3 однородная эффективная среда

$\xi_{c}, \theta$ и расчетная область (рис. 2) представлена совокупностью $N=50$ концентрических цилиндрических слоев с однородными изотропными упругими свойствами; при этом переходный цилиндрический слой с изотропными в каждой точке $\xi$ плоскости $\xi_{1} \xi_{2}$ и неоднородными по радиальной координате $\xi_{\mathrm{c}} \equiv|\xi|$ упругими свойствами $\mathbf{a}_{(\mathrm{a})}^{(0)}\left(\xi_{\mathrm{o}}\right)$ дискретизировался на $N-2$ тонких цилиндрических слоя. Дальнейшее решение аналогично подходу, использованому, например, в [1,2].

Результаты численного расчета компонент $C_{1111}^{*}$ и $C_{1212}^{*}$ тензора $\mathbf{C}^{*}$ эффективных динамических упругих свойств в плоскости изотропии $r_{1} O r_{2}$ представлены в таблице, когда круговая частота $\omega=600 c^{-1}$ и относительное объемное содержание волокон в композите $v_{F}=0,5$. Модули Юнга и коэффициенты Пуассона изотропных матрицы $E_{M}=1$ ГПа, $v_{M}=0,15$ 
и волокон $E_{F}=100 E_{M}, v_{F}=0,1$ при значении отношения $E_{M} / \rho=10^{6}$. Когда круговая частота $\omega<20 c^{-1}$, тогда численные значения компонент $C_{1111}^{*}$ или $C_{1212}^{*}$, полученные обобщенным методом самосогласования с учетом и без учета [1] динамических эффектов, различаются менее, чем на $3 \%$.

Т а б ли ц а

Эффективные динамические упругие компоненты $C^{*}$

и сечения рассеяния $\gamma^{*}$ в плоскости изотропии волокнистого композита

\begin{tabular}{|c|c|c|}
\hline \multirow{2}{*}{ Эффективные константы композита } & \multicolumn{2}{|c|}{ Результаты расчета } \\
\cline { 4 - 4 } & Статический [1] & Динамический \\
\hline$C_{1111}^{*} / C_{1111}^{M}$ & 3,09 & 1,39 \\
$C_{1212}^{*} / C_{1212}^{M}$ & 3,01 & 1,45 \\
\hline$\gamma_{1}^{*} / r_{F}^{2}$ & - & 5,99 \\
$\gamma_{2}^{*} / r_{F}^{2}$ & - & 8,84 \\
\hline
\end{tabular}

Численные значения эффективных сечений рассеяния $\gamma^{*}$ приведены в таблице для распространяющейся вдоль оси $r_{1}$ продольной $\left(\gamma_{1}^{*}\right)$ или поперечной $\left(\gamma_{2}^{*}\right)$ волн в плоскости изотропии $r_{1} O r_{2}$ однонаправленного волокнистого композита. Величина $\gamma^{*}$ рассчитана из уравнения $\gamma^{*}=E^{*} / I^{*}$, где $I^{*}$ - интенсивность падающей волны, рассеяная энергия за единицу времени [1]:

$$
E^{*}=\frac{i \omega R}{4} \int_{0}^{2 \pi}\left(\bar{\sigma}_{(a) \xi \xi} \bar{u}_{(a) \xi}+\bar{\sigma}_{(a) \xi \theta}^{\cdot} \bar{u}_{(a) \theta}-\bar{\sigma}_{(a) \xi \xi} \bar{u}_{(a) \xi}^{\cdot}-\bar{\sigma}_{(a) \xi \theta} \bar{u}_{(a) \theta}\right)_{\mid \xi_{0}=R} d \theta .
$$

Для $R>>r_{F}$, символ • обозначает комплексно сопряженные величины.

\section{БИБЛИОГРАФИЧЕСКИЙ СПИСОК}

1. Труэлл Р., Эльбаум Ч., Чик Б. Ультразвуковые методы в физике твердого тела. М.: Мир. 1972. 307 с.

2. Гузь А.Н., Кубенко В.Д., Черевко М.А. Дифракция упругих волн. Киев: Наук. думка. 1978. 308 с.

3. Пришивалко А.П., Бабенко В.А., Кузьлин В.Н. Рассеяние и поглощение света неоднородными и анизотропными сферическими частицами. Минск.: Наука и техника. 1984. 263 с.

4. Ньютон Р. Теория рассеяния волн и частиц. М.: Мир. 1969. 607 с.

5. Кравияов Ю.А., Татарский В.И. Статистические явления при дифракции волн. Рязань, 1975. 102 с.

6. Шермергор Т.Д. Теория упругости микронеоднородных сред. М.: Наука. 1977. 400 с.

7. Чернов Л.А. Волны в случайно-неоднородных средах. М.: Наука. 1975.172 с.

8. Исимару А. Распространение и рассеяние волн в случайно-неоднородных средах. В 2 т. М.: Мир. $1981.320 \mathrm{c}$.

9. Паньков А.А. Анализ эффективных упругих свойств однонаправленного волокнистого стеклопластика обобщенным методом самосогласования // Изв. РАН. МТТ, 1999. №4. С. 78-86

10. Паньков А.А. Обобщенный метод самосогласования для композитов со случайными упругими свойствами фаз составных или полых включений // Механика композиционных материалов и конструкций. 2000, №.3. С. 310-332 